\title{
Information or Evidence? Abandoning Imaginary Worlds for Blockchains in Health Technology Assessment
}

\author{
Paul C Langley, PhD, Adjunct Professor, College of Pharmacy, University of Minnesota
}

\begin{abstract}
Commentaries published in INNOVATIONS in Pharmacy over the past 2 years have made the case that, as a basis for formulary decision making, the construction of imaginary modeled worlds fails to meet the standards of normal science. As such, they should be rejected as a basis for decision-making. While their proponents argue that imaginary constructs are key sources of information for formulary decisions, the fact is that the claims made from those models are impossible to validate. Indeed, they were never intended to be validated. Claims for product performance should be presented in evidentiary terms. That is, they should be credible, evaluable and replicable. If the commitment to imaginary worlds in technology assessment is to be abandoned a key requirement is for platforms that allow claims to be assessed in real time and in a timeframe that is meaningful to decision makers. Recent developments in blockchain technology offer the prospects for platforms that meet criteria for claims assessment.
\end{abstract}

Keywords: imaginary worlds, ICER, AMCP, ISPOR, formulary decisions, blockchains

\section{Introduction}

Over the past few years the present author has published a number of commentaries in INNOVATIONS in Pharmacy that have made the case that the commitment in health technology assessment to the construction of imaginary worlds to support claims for product cost-effectiveness and pricing runs counter to the standards of normal science ${ }^{1}$. Rather than focusing on the need for an evidence based assessment of claims for pharmaceutical products and devices in target patient populations, formulary committees and other health system decision makers have been asked to rely upon information 'constructed' from imaginary worlds. This approach to decisionmaking and the consequent lack of attempts to replicate claims following product formulary approval look set to continue. A situation which is exacerbated by the role of pricing, not only the choice of a product price consistent with initial modeled claims for cost-effectiveness, but the subsequent near term abandonment of that price in favor, at least in the US, of a pricing strategy that looks to annual or semi-annual price increases to generate target revenue flows. Needless-to-say, justifications for the continued increase in product prices, which all too often exceed not only consumer price indices but also medical cost indices, are either absent or lack credibility.

The purpose of this commentary is to consider why this commitment to the construction of imaginary worlds remains a core activity in health technology assessment. Obviously, if a

Corresponding author: Paul C Langley, PhD

Adjunct Professor

College of Pharmacy University of Minnesota

Director, Maimon Research LLC

5061 North Apache Hills Trail, Tucson, AZ 85750

Email: langley@maimonresearch.com single payer health system mandates or sets guidelines for the construction of modeled imaginary worlds to support formulary decision making, a manufacturer would be foolish to object. The construction of a modeled imaginary world is far less taxing, in time and costs, compared to suggestions of a commitment to underwriting a short-term prospective observational study or pragmatic trial to evaluate or replicate claims generated for clinical outcomes and modeled costeffectiveness from pivotal Phase 3 trials. After all, there is always the risk of non-substantiation of claims. A risk, if the evidence for non-replication of trial outcomes is accepted, that is far from negligible. For a modeled lifetime cost-per-quality adjusted life year (QALY) model the question of replication is, of course, a non-starter. The claims, by construct, are nonevaluable.

In the US, the absence of a single payer system and the lack of support from government agencies in the construction of imaginary modeled worlds has not deterred professional associations such as the Academy of Managed Care Pharmacy (AMCP) and the International Society for Pharmacoeconomics and Outcomes Research (ISPOR) from recommending the construction of imaginary worlds, typically lifetime cost-perQALY models; a position that is supported by the Institute for Clinical and Economic Research (ICER) 234 . The ICER is of particular interest with the attention given to its modeled recommendations for pricing by manufacturers $\begin{array}{lll}5 & 6 & 7 \text {. The }\end{array}$ paradox appears to be that while manufacturers may criticize a specific ICER model, typically when the pricing and discounting recommendations are against the manufacturer, rather than challenge the utility of constructing imaginary worlds in the first place, the response of the manufacturer is to propose alternative and, inevitably, more favorable imaginary worlds. It is this apparent paradox and its potential resolution that will be examined here. 


\section{Absent Hypothesis Testing}

If there was any doubt as to the commitment by health technology assessment agencies to rejecting hypothesis testing of product claims in favor of imaginary constructs, the latest version of the Canadian CADTH guidelines makes this abundantly clear: Economic evaluation are designed to inform decisions. As such they are distinct from conventional research activities, which are designed to test hypotheses ${ }^{8}{ }^{9}$. The focus of the CADTH guidelines is on the construction of a cost per QALY reference case assessment of the expected costeffectiveness tradeoff of two or more interventions. The reference case is not intended to generate evaluable claims. In the framework that is presented the reference case extrapolates from currently available information. The time horizon of the reference case 'should be long enough to consider all relevant differences in the future costs and outcomes associated with the interventions being compared'. If the intervention is for a chronic disease then the lifetime of the modeled index population is the framework for the reference case. This could extend decades into the future. Expressing the decision problem in lifetime cost-per-QALY terms excludes by definition any attempts to generate evaluable claims for competing products (or even claims for the product itself). The decision problem is based, therefore, on an arbitrary construct describing the future unknown course of a disease, replete with assumptions regarding future costs, outcomes, quality of life, co-morbid conditions, compliance and mortality.

\section{Resource Misallocation and Unintended Health Outcomes}

Relying on imaginary worlds to drive formulary decisions has the potential for significant resource misallocation and unintended health outcomes that may be driven by unevaluable product claims. Unfortunately, it is no defense to argue that one-modeled imaginary world is more 'realistic' than another. Where the model extends for decades beyond the short term where modeled claims might be evaluated, providing feedback to formulary committees, we have no idea whether the modeled claims are right or even if they are wrong, and we will never know. In the absence of a commitment by a manufacturer to (i) produce evaluable claims and (ii) undertake to evaluate those claims through prospective observational studies or, in the medium to long term, agree to a registry, there is no basis for re-assessing a decision let alone evaluating the extent to which such a formulary decision has led to resource misallocation and adverse health outcomes in target patient populations.

The inability or unwillingness of manufacturers to develop and stand behind evaluable claims is made more problematic by the fragmented nature of US health data. Certainly, there are databases such as those generated from administrative claims, but these typically lack appropriate tracking and outcomes measures for clinical events. There are no data which monitor or track patients for their successive visits to their provider over the course of treatment with inputs from both the patient and the treating physician. Indeed, physician practices show little interest in generating their own data to assess aggregate response to therapy in target populations over the course of treatment, even to the extent of being able to respond to simple requests for the numbers of patients introduced to specific products or devices. Moving beyond this to implementing validated patient reported outcomes instruments to track responses, such as the Patient Global Impression of Change (PGIC) which is often found in phase 3 randomized trials, seem outside a practice's terms of reference.

\section{Belief in Modeling}

One question that has been asked, but with no satisfactory resolution, is whether formulary committees in the US and those outside of the professional fraternity of imaginary world architects, take modeled claims seriously? Do they see these claims as 'useful' information (whatever that means) or as a black box marketing device to set the stage for formulary positioning and pricing decisions. It is difficult to believe that formulary committees, faced with competing black box models for a manufacturer's products and comparators, are in a position even to evaluate the competing merits of the modeled assumptions let alone the lifetime modeling techniques that are applied. After all, compared to agencies such as NICE in the UK and the PBAC in Australia, formulary committees are unlikely to have the resources to ask for an independent assessment of competing models. Even where these resources are available with a technical support group, the likelihood is that the group has accepted the informational rather than the evidentiary role of modeling claims. Whether they are even prepared to admit that lifetime cost-per-QALY claims were never intended to be anything other than constructed 'information' is an open question.

Claims that a product, at the price determined by the manufacturer, is 'cost-effective' also lack credibility. Any price can be defended, in a constructed model, as cost-effective. This is seen in the 'our model is more realistic than your model' responses associated with ICER recommendations for price discounting under various willingness-to-pay scenarios. It is always possible to choose a price, tweak assumptions (or even provide an entirely different model structure) to support a price that falls below some willingness-to-pay threshold. It becomes a game. One imaginary construct is set against another. After all, as noted above, a price can be selected to ensure formulary acceptance ('our product is cost-effective versus comparators') followed in short order by a price increase that effectively junks the previous modeled claim. It is unusual, if not unheard of, for a formulary committee to ask for a reworking of a model after a future unilateral price increase by a manufacturer to recalibrate previous claims for comparative cost-effectiveness.

\section{Accepting Non-Evaluable Claims}

While it is possible to make the case that modeled 'for information' claims should be put to one side in favor of 
credible evidence based claims, the fact is that constructing modeled claims continues to be the mainstay of academic groups and consultants in the US, whether these claims are couched in clinical or cost-effectiveness terms. An industry has developed around the AMCP guidelines, with continuing iterations of the guidelines still hewing to the lifetime cost-perQALY paradigm. Putting to one side the obvious attraction of mathematical modeling and building of lifetime frameworks as an academic 'build your CV' exercise and the willingness of journal editors to publish these models, a reasonable question is why does this persist? The ICER continues to have an impact and continues as a non-profit group to attract funds for constructing imaginary worlds. The ICER, in an important sense, sets the stage and establishes the parameters for nonevaluable 'information' which, given its recommendations, can be attacked or supported by manufacturers.

The ICER process of engagement and its contracting with academic research centers, plus limited access granted to manufacturers to 'assess' the modeling framework, gives a seal of approval to the 'information' paradigm 10 11. This is reinforced by the support for imaginary worlds given by AMCP, ISPOR and overseas single payer health system agencies. Everyone is on board. Manufacturer critiques of an adverse ICER recommendation are seen as a minor scuffle over assumptions; a rearranging of deckchairs, rather than an interest in generating and supporting evaluable claims.

If there is no interest by manufacturers in moving to an evidence-based approach to formulary decision making, then it could be argued that if ICER did not exist it would have to be invented. ICER sets a seal of approval. It is part of a methodological guarantee that information only modeling is acceptable, regardless of the potential for adverse consequences and resource misallocation. More pertinent, perhaps, is that the focus on imaginary worlds deflects attention from the possibility of rigorous and ongoing evidence based reviews of product performance. If there is no possibility of evaluable claims then there is no basis for revisiting claims as part of disease area and therapeutic class reviews. Unless formulary committees are prepared to require manufacturers to submit evaluable product claims and underwrite protocols to assess those claims, as proposed in the Minnesota guidelines for formulary submissions decisions are made in what is virtually an evidence vacuum ${ }^{12}{ }^{13}$. At best, formulary committees are forced back on attempting to establish the external validity of typically short-term pivotal randomized trial claims. Attempts to undertake observational studies, establish registries or longer term randomized trials fall at the first hurdle: the absence of funding. The net result is that there is no feedback from physician practices on the effectiveness and comparative performance of the majority of pharmaceutical products and devices.
Putting evidence based evaluations of health technologies to one side in favor of the construction of lifetime cost-per-QALY worlds has put health technology assessment outside of the mainstream of normal science. The imaginary worlds paradigm is, for all practical purposes, a dead end. Certainly, technology assessment agencies can continue to mandate imaginary constructs for 'information' purposes. Whether this adds to our ability to assess the value new technologies bring to the process of care is doubtful.

\section{A Platform for Evidence}

If a major reason for continuing to accept the modeled or constructed information paradigm is the absence of readily accessible and low cost platforms for claims assessment, then the question is whether or not it is possible to establish such a platform (or platforms by disease area) for health care systems? What would be the incentives for such a platform? Would it be possible to bring together the various disparate data elements that characterize the US health care system? Can interoperability be guaranteed? Can the security of data be guaranteed? Who would 'own' the platform? What access protocols would be required? Who would manage the platform?

The first step towards establishing a platform would be to agree on the ownership of the platform data. Putting to one side the software appropriate to establishing a platform, it is worth noting that the Centers for Medicare and Medicaid Services (CMS) has formulated a proposed rule requiring healthcare organizations to provide patients with their health information in a universal electronic format ${ }^{14}$. The CMS vision, building on the MyHealthEData initiative, is to put patients in control of their own health data so they can decide how it is used. If it proves possible to assign property rights to patients, this is the necessary first step. Unfortunately, we can be assured there will be well financed objections from current health data vendors to any arrangement that abrogates their perceived property rights and their revenue streams. Having to secure patient permission, for example, from literally tens of millions of patients in the case of administrative claims data would be is a major undertaking.

According to CMS, establishing a platform that captures every encounter (including legacy encounters) within the health care system may reduce substantially practice administrative costs. At the same time the CMS objective is to drive innovation to create new 'tools and solutions' to support research and evidence-based guideline development. The goal is to connect 'seamlessly' all records to the patient's EMR. Whether this is practical is an open question given the plethora of EMR systems, their potential claims to property rights, and the additional need as patients move from one provider to another to transfer all records from one EMR system to another. Consideration does not appear to have been given to a platform 
outside of EMRs which the patient subscribes to for their lifetime or which can be transferred to another platform.

\section{Blockchains as Platforms}

Increasing attention is being given to the role of blockchain software as platforms for the encrypted, safe storage of personal health records. A patient would subscribe to a blockchain and request all health records be copied. This data transfer would occur in real time (with legacy records captured on initial subscription) with all successive health system encounters, including data from devices. Ownership of the blockchain data would overcome issues of interoperability of records and facilitate portability of a complete medial history.

Expecting patients to subscribe as individuals to a health blockchain is probably wishful thinking. They would have neither the time nor the skills and resources to organize record transfers, nor the skills to establish smart contracts for record access by third parties. The more likely scenario is the role of physician practice groups, specialty physician practices and health systems to encourage the adoption of blockchains by their patient population. It is this scenario that sets the stage for an evidence platform with property rights assigned to the patient. As a first step the patient medical record held by the individual practice would be copied to the blockchain. Supplementary data from referrals and other encounters such as hospitalizations and urgent care and emergency room visits could be added as these recognize property rights and links are established to the platform for data retrieval.

Even so, managing recruitment to the blockchain and the copying of health records are critical to establishing a viable platform. Management is most likely to be through the blockchain vendor. This would involve an agency contract with physician practices and health systems, with the vendor as gatekeeper to the blockchain to ensure property rights are recognized. Patients would be kept appraised of options to monetize to give access to their data by third parties. The blockchain management would provide the necessary indexing and search functions for data interrogation to identify target patient groups for third party access.

While the potential for monetizing health data has been seen as a major incentive to blockchain adoption, blockchains are also a potentially critical tool for enhancing the process of care. Capturing data in real time can allow interactions between physician and patients to assess response to therapy as well as providing the framework for assessing evaluable product and device claims. The existence of blockchain platforms would allow formulary committees to put to one side decisions based on constructed 'information' in favor of real time claims assessment capturing feedback from patients and physicians.

A blockchain platform is not a disease registry. While this may be obvious it is worthwhile noting the synergies between disease registries and a blockchain platform. If property rights are recognized then the contribution a patient or the provider on the patient's behalf makes to the registry should be captured by the blockchain. At the same time, recognizing the inherent property rights of the patient opens up the opportunity for the patient to monetize their registry membership rather than assigning their rights to the registry owner. Blockchains also allow for the recruitment to registries through their ability to identify target patient populations and assign registry membership criteria (e.g., risk stratification of patients). Once a platform is established, registries can be accommodated within the blockchain. This offers a potentially significant source of revenue to the blockchain vendor as manager as well as monetization opportunities for the patient. The added advantage, of course, is that embedding a registry within a blockchain gives access to the patient's complete medical record, supplementing and providing added value to the registry data.

\section{Conclusions}

From the perspective of health technology assessment, the ability to abandon constructed claims for comparative performance based on lifetime cost-per-QALY models is a necessary next step. The adoption of blockchain software offers this possibility. A blockchain platform has the potential to support and provide feedback in real time for claims made by manufacturers. Protocols can be developed, target patient groups identified, smart contracts exchanged and incentives provided for patient and, if required, physician engagement. This is not going to be easy. There are many vested interests focused on maintaining the present system, ranging from the health technology assessment community to data vendors. Property rights may be challenged. Healthcare systems may claim they have insufficient resources and incentives. Manufacturers may be reluctant to support the credible claims evaluation. Incentives may be misaligned with uptake of the platform. These are all obstacles to consider and to set against a significant unmet medical need in applying the standards of normal science to formulary decisions.

Conflicts of Interest: None declared 


\section{REFERENCES}

${ }^{1}$ Innovations in Pharmacy. https://pubs.lib.umn.edu/index.php/innovations/section/view/formularyevaluations

${ }^{2}$ Langley PC. Modeling imaginary worlds: Version 4 of the AMCP Format for Formulary Submissions. Inov Pharm. 2016;7(2): No. 11

${ }^{3}$ Langley PC. Validation of modeled pharmacoeconomic claims in formulary submissions. J Med Econ. 2015;18(12):993-99

${ }^{4}$ Langley PC. Resolving Lingering Problems or Continued Support for Pseudoscience? The ICER Value Assessment Update. Inov Pharm. 2017;8(4): No 7

${ }^{5}$ Langley PC. Imaginary Worlds and the Institute for Clinical and Economic Review (ICER) Evidence Report: Targeted Immune Modulators for Rheumatoid Arthritis. Inov Pharm. 2017;8(2): No. 10.

${ }^{6}$ Langley PC. Multiple Sclerosis and the Comparative Value Disease Modifying Therapy Report of the Institute for Clinical and Economic Review (ICER). Inov Pharm. 2017;8(1): No. 12

${ }^{7}$ Langley PC. Cost-Effectiveness and Formulary Evaluation: Imaginary Worlds and Entresto Claims in Heart Failure. Inov Pharm. 2016;7(3): No. 6

${ }^{8}$ Canadian Agency for Drugs and Technologies in Health. Guidelines for the economic evaluation of health technologies: Canada $4^{\text {th }}$ Ed., CADTH. Ottawa 2017

${ }^{9}$ Langley PC. True North: Building Imaginary Worlds with the Revised Canadian (CADTH) Guidelines for Health Technology Assessment. Inov Pharm. 2017;8(2): No. 9.

${ }^{10}$ Langley PC. Transparency, Imaginary Worlds and ICER Value Assessments. Inov Pharm. 2017;8(4): No 11

${ }^{11}$ Langley PC. Alternative Facts and the ICER Proposed Policy on Access to Imaginary Pharmacoeconomic Worlds. Inov Pharm. 2018;9(2): No. 10

12 Langley PC. Guidelines for Formulary Evaluation [Proposed]. Program in Social and Administrative Pharmacy. College of Pharmacy. University of Minnesota. Version 2.0. December 2016.

www.pharmacy.umn.edu/departments/pharmaceutical-care-health-systems/department-programs-and-centers

${ }^{13}$ Langley PC. Nullius in Verba: Version 2.0 of the University of Minnesota, School of Social and Administrative Pharmacy Program, Proposed Guidelines for Formulary Evaluation. Inov Pharm. 2016;7(4): No 16

${ }^{14}$ https://www.healthdatamanagement.com/news/cms-proposes-rule-to-overhaul-medicare-aco-program 